\title{
An Audit of Clinical and Sociodemographic Profile of Electroconvulsive Therapy in Kashmir
}

Mansoor Ahmad Dar ${ }^{1}$, Rayees Ahmad Wani ${ }^{1}$, Yasir Hassan Rather ${ }^{1}$, Zaid Ahmad Wani ${ }^{1}$, Arshad Hussain ${ }^{1}$, Majid Shafi Shah ${ }^{1}$, Inaamul Haq ${ }^{2}$, Khurshid Ahmad Bhat $^{3}$ and Basharat Saleem ${ }^{4}$

${ }^{1}$ Department of Psychiatry, Government Medical College, Srinagar, Jammu and Kashmir, India

${ }^{2}$ Department of Community Medicine, Government Medical College, Srinagar, Jammu and Kashmir, India

${ }^{3}$ Medical officer, Directorate Of health services Srinagar, Jammu and Kashmir, India

${ }^{4}$ Department of Anaesthesia, Government Medical College, Srinagar, Jammu and Kashmir, India

*Corresponding author: Mansoor Ahmad Dar, Department Of Psychiatry, Government Medical College, Srinagar, Jammu and Kashmir, India, Tel: 009596010111; Email: gaashmansoor@gmail.com

Received date: May 10, 2014; Accepted date: May 19, 2014; Published date: May 31, 2014

Copyright: (c) 2014 Ahmad Dar M, et al.This is an open-access article distributed under the terms of the Creative Commons Attribution License, which permits unrestricted use, distribution, and reproduction in any medium.

\begin{abstract}
Background: Patterns of electroconvulsive therapy (ECT) use and the clinical and sociodemographic profile have recently been extensively surveyed in developed world. However, data from Indian Sub-continent is limited although ECT is widely used in this part of world.
\end{abstract}

Objective: This study aimed to study the clinical and sociodemographic profile of ECT patients in Kashmir (India).

Methods: Case sheets of patients attending the lone ECT facility for last 3 years were analyzed retrospectively.

Results: There were 90 patients who received 691 ECT treatments in three years. Majority 64.5\% (58) were males and were from rural background $58.8 \%$ (53). The average number of treatments per patient was 7.7 . Major depression was seen $27.8 \%$ (25). Schizophrenia and Obsessive Compulsive Disorder was seen in 14.5\% (13) each. Overall affective disorders formed the major group. Overall 44 of 63 (69.84\%) showed an improvement.

Conclusions: This was the first such study from this part of Indian sub-continent. The provision of ECT in only one centre is clearly insufficient for such a large study population. With the overall limited availability of the facility the protocol of the ECT services here are in line with the global trend. Affective disorders formed the main chunk in contrast to rest of India and OCD had a significant representation which is an area of research.

Keywords: Electroconvulsive therapy; Kashmir; Affective disorders; OCD

\section{Introduction}

After its introduction in 1938 in Italy, the use of electroconvulsive therapy (ECT) expanded during the 1940s [1]. It is the oldest method of somatic treatment, long before chlorpromazine and lithium came. After the initial surge, the use of ECT declined with advent of psychotropics, however its use picked up momentum after 1980s for its utility in drug resistant cases [2,3].

Although originally used in schizophrenia, presently ECT is considered an effective and relatively safe treatment of severe psychiatric states, including major depression, mania, and schizophrenia $[4,5]$. In certain situations, for example, treatmentresistant schizophrenia, ECT augmentation is still the treatment of choice [6]. Overall the use in affective disorders has been the main indication of ECT [7].

ECT is being prescribed worldwide, around one million patients being treated annually [8]. ECT standards and practice vary markedly between different countries, within same country, and even within individual centers [9]. Several studies have examined the practice of ECT in Asian countries where psychotic and affective disorders predominate the list and a wide variation in usage and clinical profile [10]. Chung while reporting ECT use in Hong Kong in a 12-month period observed that major depressive disorder was the most common diagnostic indication (40\%), followed by schizophrenia (23\%), bipolar disorder, manic or mixed episode (19\%), bipolar disorder, depressed episode (10\%), and schizoaffective disorder (9\%) [11].

Kashmir Valley has a special geopolitical place and the data reveals that in the conflict situation over the past two decades, there has been a phenomenal increase in psychiatric morbidity in Kashmir and so the need for psychiatric services [12]. The application of ECT has been one the main pivots of psychiatric services in Kashmir, but no study to date has systematically attempted to obtain data on all ECT treatments within such a large population base. With this overview in mind this study was conducted in the lone Institute of the Valley with ECT facility available. A methodological limitation of the study is that it is retrospective and reviewed data that was available at the institute. 
Citation: Ahmad Dar M, Ahmad Wani R, Hassan Rather Y, Ahmad Wani Z, Hussain A, et al. (2014) An Audit of Clinical and Sociodemographic Profile of Electroconvulsive Therapy in Kashmir. J Psychiatry 17: 129. doi:10.4172/2378-5756.1000129

Page 2 of 5

\section{Method}

The study was carried at Department of Psychiatry, Government Medical College Srinagar, Jammu and Kashmir, India which is a major psychiatric facility. The hospital has a well maintained ECT case sheets which contain the basic soicodemographic and clinical details of the patients attending for ECT. The data of patients attending ECT from January 2011 to December 2013 was collected, reviewed and analysed systematically. Diagnosis was the principle diagnosis for which ECT was given.

\section{Results}

The ECT facility at Department of Psychiatry, Government Medical College Srinagar, Jammu and Kashmir, India is the only such facility catering to the needs of people of whole Kashmir Valley, Ladakh and parts of Jammu Division Comprising a population of 6,907,623 [13] spread over approximately 22,236 square kilometres [14].

Modified bilateral ECT was administered twice a week and the patients are recruited both from IPD and OPD. A formal informed consent was taken for every ECT treatment. A resident Psychiatrist administered the ECT with the aid of an anaesthesiologist and an assistant. It was supervised by a consultant psychiatrist. An indigenously manufactured brief-pulse, constant energy machine (Medicaid Systems, Chandigarh, India) was used with a constant current of $1 \mathrm{~m} \mathrm{~A}$ and a pulse width of one millisecond. Frequency and duration of current was adjusted as per the dose needed. Motor seizure was regularly monitored by the cuff method. Propofol and succinylcholine was routinely used along with other pre medications, thiopental was used as a second line agent. ECT was administered in a separate ECT suite with a three bedded recovery room. In the ECT suite there was a monitor for vitals with an electrocardiogram, an anaesthetic apparatus, a defibrillator, oxygen equipment, pulse oximeter, suction apparatus, defibrillator, and the necessary emergency drugs.

From January 2011 to December 2013 there were 90 patients who received 691 ECT treatments (Table 1). The average number of treatments per patient was 7.7 (standard deviation 2.12) with the shortest course of one treatment and the longest one of twenty-six treatments. Crude ECT usage rate expressed as the number of patients treated per 100,000 resident population per annum was 0.44 . Majority $64.5 \%$ (58) were males and a sizable number of patients were from rural background $58.8 \%$ (53). Majority $72.2 \%$ (65) represented the adult age group of less than 40 years. Two patients were pregnant, both in the second trimester. 12 patients received more than one treatment course with one of them receiving four treatment courses during these three years.

The diagnosis of these patients varied from major depression to mutism of schizophrenia at one end and neuropsychiatric manifestations of organic syndromes like systemic lupus erythematosis at the other end. Major depression was the single individual diagnosis of these patients, $27.8 \%$ (25).The average number of treatments per patient of major depression was 8.08 .

\begin{tabular}{|c|c|c|}
\hline & No of patients $(\mathrm{N}=90)$ & Percentage \\
\hline \multicolumn{3}{|l|}{ Sex } \\
\hline Males & 58 & 64.5 \\
\hline Females & 32 & 35.5 \\
\hline \multicolumn{3}{|l|}{ Domicile } \\
\hline Rural & 53 & 58.8 \\
\hline Urban & 37 & 41.2 \\
\hline \multicolumn{3}{|l|}{ Age } \\
\hline $10-24$ & 26 & 28.9 \\
\hline $25-39$ & 39 & 43.3 \\
\hline $40-54$ & 19 & 21.2 \\
\hline $55-69$ & 6 & 6.6 \\
\hline \multicolumn{3}{|c|}{ Marital status } \\
\hline Married & 54 & 60.0 \\
\hline Unmarried & 36 & 40.0 \\
\hline \multicolumn{3}{|c|}{ No of treatment courses } \\
\hline 1 & 78 & 86.7 \\
\hline 2 & 8 & 8.8 \\
\hline 3 & 3 & 3.3 \\
\hline
\end{tabular}




\begin{tabular}{|l|l|l|}
\hline 4 & 1 & 1.2 \\
\hline Diagnosis & 13 & 14.5 \\
\hline Schizophrenia & 25 & 27.8 \\
\hline Major depression & 13 & 14.5 \\
\hline Obsessive compulsive disorder & 7 & 7.8 \\
\hline Bipolar depression & 11 & 12.2 \\
\hline Mania & 16 & 17.7 \\
\hline Suicidality & 5 & 5.5 \\
\hline Others & & \\
\hline Medication status & 34 & 37.78 \\
\hline Antipsychotics & 61 & 67.78 \\
\hline Antidepressants & 32 & 35.56 \\
\hline Mood stabilisers & 71 & 78.89 \\
\hline Benzodiazepines & & \\
\hline
\end{tabular}

Table 1: Sociodemographic and clinical characteristics.

Schizophrenia and Obsessive Compulsive Disorder were represented equally, $14.5 \%$ (13). Two patients of catatonic schizophrenia also received ECT and the rest of them were mainly paranoid schizophrenia. The average number of treatments per patient of OCD was 12.2, which was highest among all. 18 Bipolar Affective Disorder (BPAD) patients received ECT, 11 of them in mania and 7 in depressive phase of the illness. The average number of treatments per patient of BPAD was 5.2, which was the lowest. 16 patients received ECT for suicidality in the form of a recent suicidal attempt, suicidal plans/wishes at the time of assessment or were the team believed that the patient had a risk of suicide. This group included the patients of intended suicide regardless of the primary diagnosis. Majority of them had depressive illness and a number of them had psychotic illnesses.

'Others' (5.5\% N=5) included patients of SLE, Parkinsonism, noncatatonic mutism of schizophrenia and schizoaffective disorders. Overall the reasons for ECT included non-response to pharmacotherapy, the severity and urgency (suicidality) of symptoms, distress to await drug response, patient preference and previous response.

The most common side effect was memory problems, which was found in $46.7 \%$ (42) patients. Headache and muscle pain was reported by $34.5 \%$ (31) and $18.9 \%$ (17) patients respectively. Post-ictal delirium was reported in two patients. No mortality was reported.

Majority of these patients were on a combination of drugs (78.89\%). Benzodiazepines were most frequent prescribed drugs, common being clonazepam, lorazepam and alprazolam. Antidepressants included a fairly equal proportion of older and newer ones. Divalprox sodium was the frequently prescribed mood-stabiliser (17 patients) while as lithium was being prescribed in 10 patients. Other mood-stabilisers included carbazepine, lamotriagine and a combination of these. Antipsychotics including both typical and atypical were prescribed in 34 patients $(37.78 \%)$.
The outcome of the ECT courses could not be ascertained fully as the records didn't reveal any specific rating scale applied except for Clinical Global Impressions (CGI) scale in case of 63 patients only. Out of this limited information it was observed that 36 out of 63 patients reported a major improvement at the end of course (read as Much Improved on CGI-I) while as 10 of them reported no improvement (No Change on CGI-I) and 8 of them read as Very Much Improved. Number of patients reporting being Minimally Improved and Minimally Worse on CGI-I was 9 and 2 respectively. Overall 44 of 63(69.84\%) showed an improvement. Maximum improvement was seen in suicidality, bipolar affective disorders, major depression and the least improvement was reported in OCD and schizophrenia.

\section{Discussion}

This is the first retrospective audit study from Northern India. Since this study is carried at the only and the major Psychiatric facility in Kashmir Valley, it is likely to give a clear view of ECT practices and utilization in this part of world. Since it is more like an audit and not a simple survey, the results depicted are very near to the actual practice of ECT, although the retrospective nature of the study is a shortcoming.

Our finding of ECT usage rate of 0.44 per 10000 population in Kashmir Valley is far less than the worldwide varying rates of 1.1 in Poland to 51 in United States [15,16]. In Asia, ECT usage rate has been only reported from Thailand (11.5) and Hong Kong ranging (2.7-3.4) $[11,17]$. Although the ECT utilization rate has not been reported from rest of India for comparisons at the local level, our rate is still far less than the global trend. Explanations to this could be complex, ranging from the availability of services, professional beliefs concerning the efficacy and safety of ECT, the socio-cultural factors and the stigma [18]. Whatever may be the reason our finding presses for the need to 
develop such facilities, educate the people, and update the clinicians and to expand the already available services.

Average number of treatments was hovering around the worldwide average of 8 treatments per patient. The Asian average has been 6-8 treatments per patient [7]. The closeness of values is a rough predictor of adherence to global norms/guidelines by our institute. However the wide range of ECT treatments per patient depicts the variable clinical profile of the patients. A good number of patients had more than one treatment course, which on one hand depicts the cycling nature of illnesses and the limited time-bound effect of ECT but on the other hand points towards the belief of these patients on this neurostimulative technique. However this cannot be concluded without further research.

The predominance of males has been as per the global and the national trend $[19,20]$. More than seventy percent of our patients were aged less than forty years which is different than western literature $[8,21]$. However similar pattern of age distribution has been reported in Thailand.17 The lower age distribution may represent the different symptom profile of Asian patients. Moreover the preferences of the psychiatrist and anaesthesiologist to selectively exclude elderly with medical comorbities and the maintained family structure in this part of the world leading to less severe illnesses in elderly could be contributing for their decreased representation for ECT.

Affective disorder (unipolar/bipolar depression) was the main diagnoses in our study as has been seen in Australia and New Zealand [21-23]. Affective disorders have been also the main diagnoses in USA (72-92\%) [16,24]. Main diagnostic indication in Asia overall has been schizophrenia [17]. However, in Saudi Arabia [25], Pakistan [26], and Hong Kong [27], depressive illness was the main indication (over $60 \%)$, similar to our observation. The lesser use of ECT in schizophrenia (14.5\%) could be due to doubts about the co-operativity of the patient and lesser patient preference for ECT. Moreover the continuous use and free availability of long-acting antipsychotics for last four years in this institution could be reason for decreased need for ECT in schizophrenics. The encouraging results in affective disorders further might have led to deputation of more such patients for ECT.

The percentage of OCD (14.5\%) was much more than what has been studied so far which delineates the overall prolonged course and refractoriness of this disorder to other therapies [10]. The overrepresentation of OCD in our study points towards a relatively better outcome on ECT which opens a new horizon of research in the management of OCD. Although the response was not as promising as in affective disorders but was definitely promising for further work in this area.

Sucidality being an emergency to both the clinician and the caregivers represented significantly, depicting the rapid action and acceptability of ECT in such situations. Most of these suicidal patients showed a rapid and significant change on CGI-I, depicting the underlying affective illness as observed and reported in literature [28].

The response to ECT by majority of patients is consistent, as the chunk of patients belonged to affective disorders and as would be expected from the evidence elsewhere in literature, patients with affective disorders were the major responders [29,30]. Decreased response by schizophrenics is presumed to be the effect of deputation of more severe and resistant cases for ECT. The response and percentage of OCD patients is a new horizon as already discussed. However the response to ECT can't be contested upon as a single subjective scale was administered that too by different persons.
Further there was no information about the patients who completed the treatment course and who didn't. Further the follow-up post ECT was not available to give us a better idea of overall efficacy.

The rate of adverse event being lesser than in literature, although seems promising but the trend of underreporting can't be denied [31]. Also there was no use of rating scales which precisely document the expected complications.

It was heartening to find that only bilateral ECT was used, fitting well with the convincing evidence of bilateral ECT being efficacious than unilateral. Further the administration of two ECTs per week is in unison with the recommendations of Royal College of Psychiatrists [32]. Further the team approach, coordination and uniformity of protocol, well equipped and well monitored ECT suite, and use of approved brief-pulse machine was a promising sign. However administration of ECT in a bigger ECT room with application of rating scales would be warranted in future.

\section{Conclusion}

It is satisfactory to note that ECT services in this part of India are in line with global trends, although more such facilities need to come up. The very limited availability of this effective treatment modality be addressed by the state health authorities. Overall affective disorders form the chunk of patients receiving ECT, and OCD has a significant representation. The non-response to drugs and urgency/severity of symptoms are the main reasons of ECT. Moreover prospective research is needed to evaluate the efficacy of ECT in different disorders.

\section{Competing Interests}

The authors declare that they have no competing interest.

\section{Authors' Contributions}

MAD, BS managed data collection. MAD, KAB and RAW wrote the first draft of manuscript. YHR, ZAW and AH performed analysis. MSS, IH and AH did the proof reading. All authors contributed to design, interpretation and paper writing, and approved the final version of manuscript.

\section{References}

1. Abrams R (2002) History of ECT. In: Electroconvulsive Therapy. 4th ed. New York: Oxford University Press, USA.

2. McCall WV (2001) Electroconvulsive therapy in the era of modern psychopharmacology. Int J Neuropsychopharmacol 4: 315-324.

3. American Psychiatric Association. 2001. Pp. 355 in R. D.Weiner, ed. The Practice of electroconvulsive therapy: recommendations for treatment, training, and privileging: a task force report of the American Psychiatric Association. The American Psychiatric Association VII,Washington, USA.

4. American Psychiatric Association. The Practice of ECT: Recommendations for Treatment, Training and Privileging. 2nd ed. Washington, DC.

5. Royal College of Psychiatrists (1995) The ECT Handbook. The 2nd Report of the Royal College of Psychiatrists_Special Committee on ECT. Council Report CR39. London, UK.

6. Fink M, Sackeim HA (1996) Convulsive therapy in schizophrenia? Schizophr Bull 22: 27-39. 
Citation: $\quad$ Ahmad Dar M, Ahmad Wani R, Hassan Rather Y, Ahmad Wani Z, Hussain A, et al. (2014) An Audit of Clinical and Sociodemographic Profile of Electroconvulsive Therapy in Kashmir. J Psychiatry 17: 129. doi:10.4172/2378-5756.1000129

Page 5 of 5

7. Leiknes KA, Jarosh-von Schweder L, Høie B (2012) Contemporary use and practice of electroconvulsive therapy worldwide. Brain Behav 2: 283-344.

8. Prudic J, Olfson M, Sackeim HA (2001) Electro-convulsive therapy practices in the community. Psychol Med 31: 929-934.

9. Glen T, Scott AI (2000) Variation in rates of electroconvulsive therapy use among consultant teams in Edinburgh (1993-1996). J Affect Disord 58: 75-78.

10. Chanpattana W, Kramer BA, Kunigiri G, Gangadhar BN, Kitphati R, et al. (2010) A survey of the practice of electroconvulsive therapy in Asia. J ECT 26: 5-10.

11. Chung KF (2003) Electroconvulsive therapy in Hong Kong: rates of use, indications, and outcome. J ECT 19: 98-102.

12. Margoob MA (1995) A study of present magnitude of psychiatric disorders and the existing treatment services in Kashmir (1990-1994). JK Practitioner 2:165-168.

13. Government of India, ministry of home affairs.

14. Government of India, ministry of home affairs.

15. Gazdag G, Palinska D, Kloszewska I, Sobow T (2009) Electroconvulsive therapy practice in Poland. J ECT 25: 34-38.

16. Rosenbach ML, Hermann RC, Dorwart RA (1997) Use of electroconvulsive therapy in the Medicare population between 1987 and 1992. PsychiatrServ 48: 1537-1542.

17. Chanpattana W, Kramer BA (2004) Electroconvulsive therapy practice in Thailand. J ECT 20: 94-98.

18. UK ECT Review Group (2003) Efficacy and safety of electroconvulsive therapy in depressive disorders: a systematic review and meta-analysis. Lancet 361: 799-808.

19. Chanpattana W, Kunigiri G, Kramer BA, Gangadhar BN (2005) Survey of the practice of electroconvulsive therapy in teaching hospitals in India. J ECT 21: 100-104.

20. Teh SP, Xiao AJ, Helmes E, Drake DG (2005) Electroconvulsive therapy practice in Western Australia. J ECT 21: 145-150.

21. Baghai TC, Marcuse A, Möller HJ, Rupprecht R (2005) Electroconvulsive therapy at the Department of Psychiatry and Psychotherapy, University of Munich. Development during the years 1995-2002. Nervenarzt 76: 597-612.

22. Wood DA, Burgess PM (2003) Epidemiological analysis of electroconvulsive therapy in Victoria, Australia. Aust N Z J Psychiatry 37: 307-311.

23. O'Dea JF, Mitchell PB, Hickie IB (1991) Unilateral or bilateral electroconvulsive therapy for depression? A survey of practice and attitudes in Australia and New Zealand. Med J Aust 155: 9-11.

24. Scarano VR, Felthous AR, Early TS (2000) The state of electroconvulsive therapy in Texas. Part I: reported data on 41,660 ECT treatments in 5971 patients. J Forensic Sci 45: 1197-1202.

25. Alhamad AM, al-Haidar F (1999) A retrospective audit of electroconvulsive therapy at King Khalid University Hospital, Saudi Arabia. East Mediterr Health J 5: 255-261.

26. Naqvi H, Khan MM (2005) Use of electroconvulsive therapy at a university hospital in Karachi, Pakistan: a 13-year naturalistic review. J ECT 21: 158-161.

27. Chung JPY, Yim PHW, Dunn ELW (2009) Clinical and treatment characteristics of Chinese patients undergoing electroconvulsive therapy in an acute psychiatric unit in Hong Kong. Hong Kong J. Psychiatr 19: 150-154.

28. Frankel FH (1984) The use of electroconvulsive therapy in suicidal patients. Am J Psychother 38: 384-391.

29. Husain MM, Rush AJ, Fink M, Knapp R, Petrides G, et al. (2004) Speed of response and remission in major depressive disorder with acute electroconvulsive therapy (ECT): a Consortium for Research in ECT (CORE) report. J Clin Psychiatry 65: 485-491.

30. Medda P, Perugi G, Zanello S, Ciuffa M, Cassano GB (2009) Response to ECT in bipolar I, bipolar II and unipolar depression. J Affect Disord 118: 55-59.

31. Chanpattana W (2007) A questionnaire survey of ECT practice in Australia. J ECT 23: 89-92.

32. Dunne RA, Mcloughlin DM (2005) ECT prescribing and practice. In: The ECT Handbook: The Third Report of the Royal College of Psychiatrists' Special Committee of ECT. RCPsych Publications 128: 28-44. 